\title{
A Study of Etiopathology and Management of Facial Palsy at a Tertiary Care Institute
}

\author{
${ }^{1}$ Dr. G. Sreekanth, ${ }^{2}$ Dr. Sravan Kumar Ch., ${ }^{3}$ Dr. Naveed Ahmed, \\ ${ }^{4}$ Dr Pavan Kumar, \\ ${ }^{I}$ Asst.professor, Department of ENT, Head \& Neck Surgery, Gandhi Medical College, Secunderabad. \\ ${ }^{2}$ Senior Resident, Department of ENT, Head \& Neck Surgery, Gandhi Medical College, Secunderabad. \\ ${ }^{3}$ Professor and HOD, Department of ENT, Head \& Neck Surgery, Gandhi Medical College, Secunderabad. \\ ${ }^{4}$ Junior Resident, Department of ENT, Head \& Neck Surgery, Gandhi Medical College, Secunderabad.
} $M B B S . M S-E N T$

\begin{abstract}
The causes and management of Facial nerve paralysis have been well documented in the literature. Facial expression is an important means of social communication in human beings. Facial nerve dysfunction can dramatically affect a patient's quality of life. It causes both psychological and Emotional trauma. A prospective study was conducted at a tertiary ENT care institute over a period of 3 years to identify various causes of facial nerve palsy and to analyse improvement in terms of grades. Traumatic facial palsy was more common in male patients in our study. The incidence of facial palsy was highest in age group of 21-40 yrs with 21 cases [42\%]. Trans-mastoid approach is an ideal approach for decompression of facial nerve in cases with pathology at the level of horizontal, $2^{\text {nd }}$ genu and vertical segments.
\end{abstract}

Keywords: Facial nerve palsy, Facial paralysis, Facial nerve decompression, Bell's palsy, Traumatic facial palsy.

\section{Introduction}

Facial nerve (CN-VII) is one of the unique cranial nerves innervating several tiny muscles and maybe the only one travelling through a long bony tunnel.Facial expression is an important means of social communication in human beings.In 1821, Sir Charles Bell, an anatomist and a surgeon first described facial palsy and named it "Bell's Palsy'." Facial nerve dysfunction can dramatically affect a patient's quality of life. It causes both psychological and Emotional trauma.

\section{Aims and Objectives}

The aims of the study are to identify various causes of facial nerve palsy presenting to a tertiary care institute over a period of 3 years, to analyse improvement in grade of facial palsy after treatment depending on etiology of palsy and to correlate the improvement in facial nerve function with the cause of the facial palsy.

\section{Materials and Methods}

A prospective study was conducted at a tertiary ENT care institute over a period of 3 years i.e. from July 2012 to May 2015 that included 50 cases of facial nerve palsy of various etiologies.Cases with a minimum of grade III facial nerve palsy are selected and included in the study. Patients of all age group and both sexes are included.Patients who cannot be followed up for the minimum stipulated time were excluded from the study.

The study included collection of a detailed history, clinical examination, identification of cause of facial palsy, grading of facial palsy according to House-Brackmann staging ${ }^{2}$, details of surgical procedure, intraoperative findings and 6 month follow-up.Haematological, Audiological, Radiological, Topodiagnostic investigations are conducted in every case. The severity of facial nerve paralysis is graded based on HouseBrackmann Grading Scale.Follow-up of cases was done at regular intervals of 1 week, 3 weeks, 6 weeks, 3 months and 6 months. The data is collected, analysed and statistically presented.An achievement of grade III or below after 6 months follow-up following facial nerve decompression was taken as a positive outcome.Facial nerve monitoring was not used routinely as it has not been the practice at our institute.

\section{Results}

Out of the total 50 cases included in the study, 33 are male [66\%] and 17 are female [34\%]. Casesare arranged into age groups 0-20yrs -10 cases, $21-40 \mathrm{yrs}-21$ cases, $41-60 \mathrm{yrs}-17$ cases, $>60 \mathrm{yrs}-2$ case.The incidence of facial palsy was highest in age group of 21-40 yrs with 21 cases [42\%]. The reason why adults are more commonly effected is because traumatic cases are seen more in adults. Majority of cases of traumatic 
facial palsy included in the study are within the age group of 21-40yrs. The number of caseswith left facial palsy are $26[52 \%]$ and right facial palsy are 24 [48\%]this is of no significance.

The causes of facial palsies[fig:1]areChronic suppurative otitis media[CSOM] in 15 cases [30\%], Trauma in 14 cases [28\%],Bell's Palsy in 8 cases [16\%], Iatrogenic in 6 cases [12\%] and others in 7 cases[14\%]. Other causes included glomus jugulare, middle ear malignancy, malignant otitis externa, acute suppurative otitis media etc. Cases are grouped according tograding of facial palsyboth at the time of presentation and after 6 months of follow-up as shown in fig:2.

15 casesof CSOM causing facial palsy and 14 cases of traumatic facial palsy were taken up for surgery. The trans-mastoid approach was used in all patients. Canal wall down mastoidectomy has been performed by the standard method in all cases included in the study except one where a intact canal wall technique was done and facial nerve was approached via posterior tympanotomy. During surgery the course of the facial nerve has been identified in all cases. Where an erosion due to cholesteatoma was found, the nerve underwent decompression a few mm proximal and distal to the site of erosion. Where the site of injury could not be made out, the entire mastoid and tympanic portion was decompressed.

Trans-mastoid approach was chosen because it is basically an ENT procedure and it maintains serviceable hearing. Most of the pathology in cases of CSOM islocated in horizontal, $2^{\text {nd }}$ genu and vertical segments which can be easily approached by this approach. All patients were explained about the possible surgical complications and the limited reversibility of good facial nerve function.

Cases of Bell`s palsy were treated conservatively with steroids and anti-virals.

\section{Discussion}

The anatomy of the facial nerve is among the most complex of the cranial nerves. A solid understanding of its complex anatomy and physiology is crucial to the surgeon's ability to both diagnose and treat disorders of the facial nerve.Common etiological factors associated with facial palsy include Bell's palsy, CSOM, Trauma, Iatrogenic, Idiopathic etc ${ }^{3}$.

Clinical Features include change in the appearance of the face, difficulty closing one eye, difficulty making expressions, difficulty with fine movements of the face, paralysis of one side of the face, difficulty eating, face feels pulled to one side etc ${ }^{3}$. Topognostic tests are intended to determine the level of facial nerve injury by testing peripheral facial nerve function. They include Schirmer's test, Stapedial reflex, electrogustometry and Submandibular salivary flow test. Electrophysiologic tests include Minimal nerve excitability test, Maximal stimulation test (MST), Electroneuronography (ENoG) and Electromyography (EMG). Non-Surgical Measures for treatment of facial nerve palsy include Steroid therapy, Antiviral therapy, Physiotherapy and Electrical stimulation.Surgical approaches commonly used are Transmastoid approach and Middle cranial fossa approach ${ }^{4,5,6}$.

According to Jin $\mathrm{Kim}^{7}$, et al. CSOM causing facial nerve paralysis is most frequently due to cholesteatoma. The presence of cholesteatoma decreased the effectiveness of surgical gain and indicated a poor prognosis after surgery. Osteitis, bone erosion, external compression, edema and inflammation of the nerve are some of the proposed etiologic factors in CSOM causing facial nerve paralysis.

All the traumatic cases included in the study were of longitudinal type of temporal bone fractures.

According to $\mathrm{Jin} \mathrm{Kim}^{7}$, et al. there is evidence of poor recovery of the facial nerve function in the patient with transverse fracture, where a middle cranial fossa approach would have been the better approach.Hemanth Chopra $^{8}$ in his study of 48 cases of facial paralysis, found that the incidence of longitudinal fracture was $51 \%$ and transverse fracture was $19 \%$.

In the present study after 6 months follow-up, in cases of CSOM with facial nerve palsy, improvement of a single grade was noted in 8 cases [16\%] and 2 grades in 5 cases [4\%]. In cases of trauma causing facial nerve palsy, improvement of a 2 grades was noted in 9 cases [18\%] and 3 grades in 2 cases [4\%].It can be concluded from this study that facial nerve decompression in cases of CSOM with facial nerve palsy is primarily aimed at clearance of disease (cholesteatoma) and prognosis is poor when compared to decompression in cases of facial nerve palsy due to trauma.

In our study, the outcome of surgical intervention was closely related to the duration of onset to surgery. Longer durations can cause more severe deterioration of the facial nerve and poor surgical outcomes. Patients with facial paralysis due to CSOM should be operated on as early as possible, regardless of the severity of facial function, the presence of cholesteatoma, type of onset, age and any previous otologic surgical history.Ikeda, et al. ${ }^{9.10}$ reported that with regard to the duration of onset of facial paralysis to surgery, the surgical timing had been thought to be an important factor.But according to Galip Zihni SANUfi et al. ${ }^{11}$ late facial nerve decompression may have still beneficial effects in patients who could not be operated on early. Early decompression of the facial nerve in case of acute paralysis has beneficial effects, but facial nerve decompression even 4 months after the onset of FNP can still have a beneficial effect, depending on the ENoG or EMG, in patients who could not be operated on early. 
The House-Brackmann scale ${ }^{2}$ is used in the study as it is the most widely used clinical instrument for facial nerve functional evaluation. Though it is used in the study we encountered difficulties regarding interobserver based variations in grading of facial nerve palsy. A more robust scale with better variables is needed.Electrodiagnostic studies may add additional information about the type and severity of injuries, thus allowing prognostic inferences.According to Cawthorne et al. ${ }^{12}$ with nerve conduction tests it is possible to predict which patients may recover quickly and completely and which slowly and not fully.

\section{Conclusion}

The conclusions are that traumatic facial palsy was more common in male patients in our study and road traffic accidents are the most common cause of traumatic facial palsy. Theincidence of facial palsy was highest in age group of 21-40 yrs. CSOM causing facial nerve paralysis is most frequently due to cholesteatoma.Trans-mastoid approach is an ideal approach for decompression of facial nerve in cases with pathology at the level of horizontal, $2^{\text {nd }}$ genu and vertical segments.

Facial nerve decompression in cases of CSOM with facial nerve palsy is primarily aimed at clearance of disease (cholesteatoma) and prognosis is poor when compared to decompression in cases of facial nerve palsy due to trauma.Patients with facial paralysis due to CSOM should be operated on as early as possible.

\section{References}

[1] D S Grewal, Bells palsy, Atlas of Surgery of the Facial Nerve, Second Edition.

[2] House JW, Brackman DE. Facial nerve grading system.Otolaryngology and Head and Neck Surgery. 1985; 93:146-7.

[3] Thanos Bibas, Dan Jiang And Michael J Gleeson, Disorders of the facial nerve, 241c, scott brown, $7^{\text {th }}$ edition.

[4] I P Tang, MS, S C Lee, MS, S Shashinder, MS, R Raman, MS,Outcome of Patients Presenting with Idiopathic Facial Nerve Paralysis (Bell's Palsy) in a Tertiary Centre - A Five Year Experience, Med J Malaysia Vol 64 No 2 June 2009155.

[5] Osma U, Cureoglu S, Hosoglu S. The complications of chronic otitis media: report of 93 cases. J Laryngol Otol. 2000;114 (2):97.

[6] Yetiser S, Tosun F, Kazkayasi M. Facial nerve paralysis due to chronic otitis media. Otol Neurotol. 2002;23(4):580.

[7] Jin Kim,Gu-Hyun Jung, See-Young Park, and Won Sang Lee, Facial Nerve Paralysis due to Chronic Otitis Media: Prognosis in Restoration of Facial Function after Surgical Intervention, Yonsei Med J 53(3):642-648, 2012.

[8] Hemanth C, Khurana AS, Munjal M, Vanitha C, Anju M, Sobti MK. Facial nerve paralysis in head injury. Indian Journal of Otology, 2002;8:86-89.

[9] Ikeda M, Abiko Y, Kukimoto N, Omori H, Nakazato H, Ikeda K. Clinical factors that influence the prognosis of facial nerve paralysis and the magnitudes of influence. Laryngoscope. 2005;115(5):855.

[10] Ikeda M, Nakazato H, Onoda K, Hirai R, Kida A. Facial nerve paralysis caused by middle ear cholesteatoma and effects of surgical intervention. Acta Otolaryngol. 2006;126(1):95.

[11] Galip Zihni SANUfi, Necmettin TANRIÖVER Taner TANRIVERD,Mustafa UZAN, Late Decompression in Patients With Acute Facial Nerve Paralysis After Temporal Bone Fracture, Turkish Neurosurgery 2007, Vol: 17, No: 1, 7-12

[12] Cawthorne, T. E.: Indication for intratemporal facial nerve surgery, Arch. Otolaryngol., 78:429-434, October 1963.

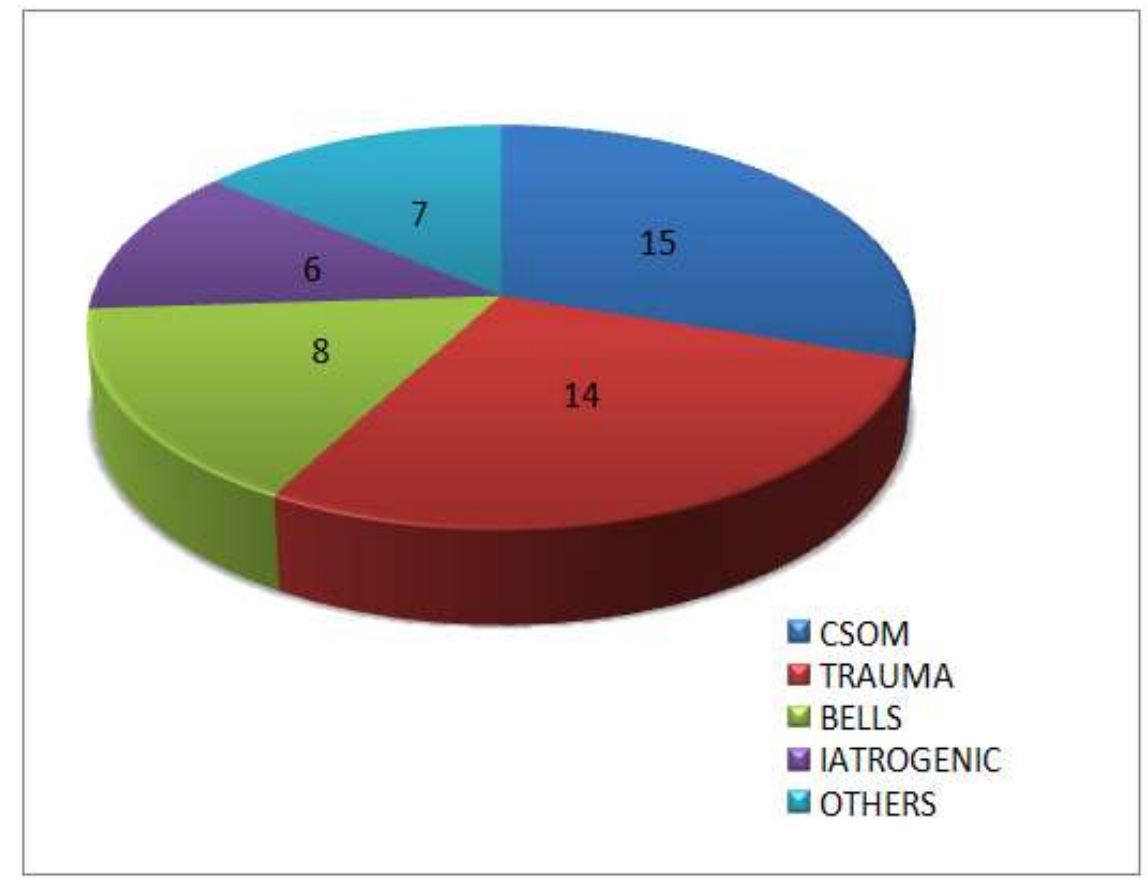

Figure 1- Pie diagram showing Etiology of facial nerve palsy 


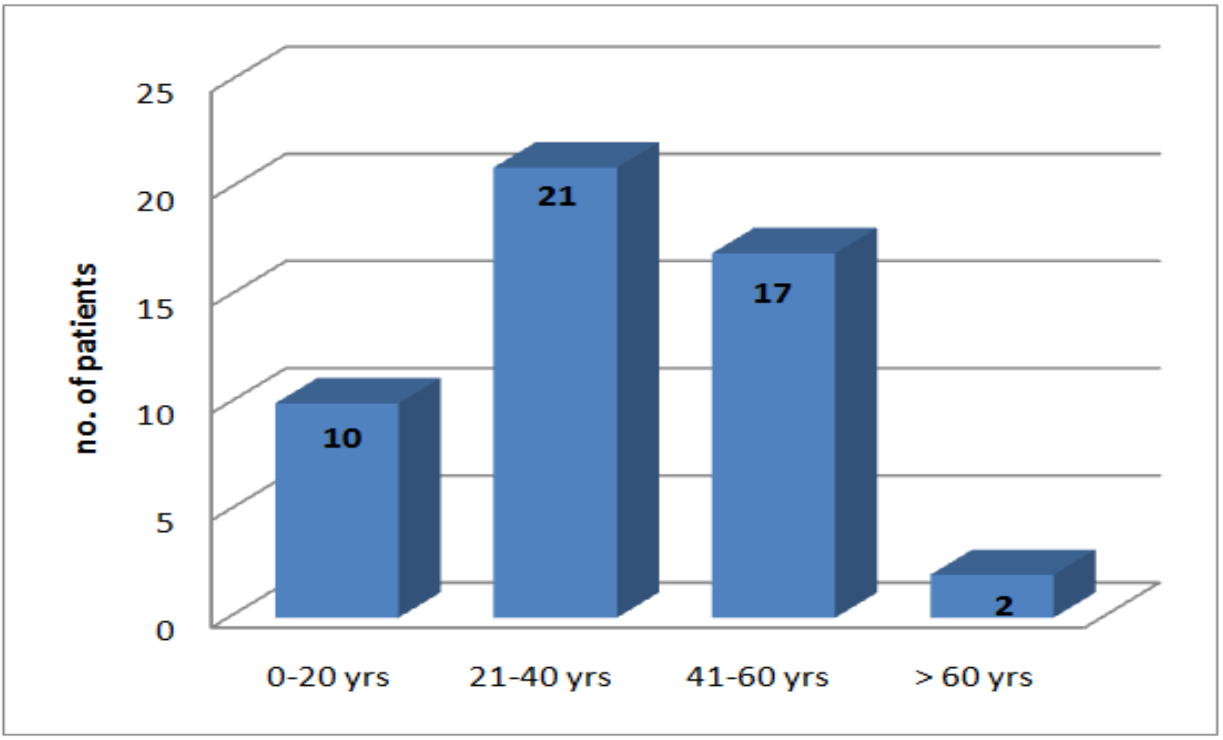

Figure 2 - Bar chart showing age distribution

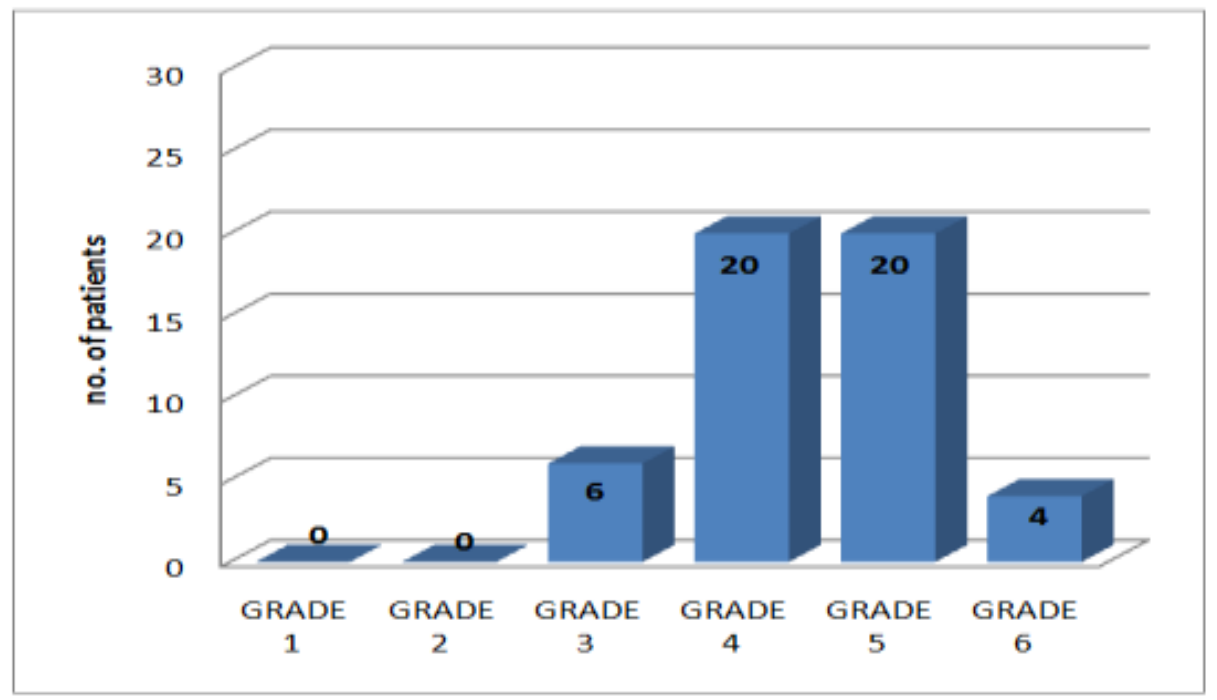

Figure 3 - Bar chart showing pre-operative grading

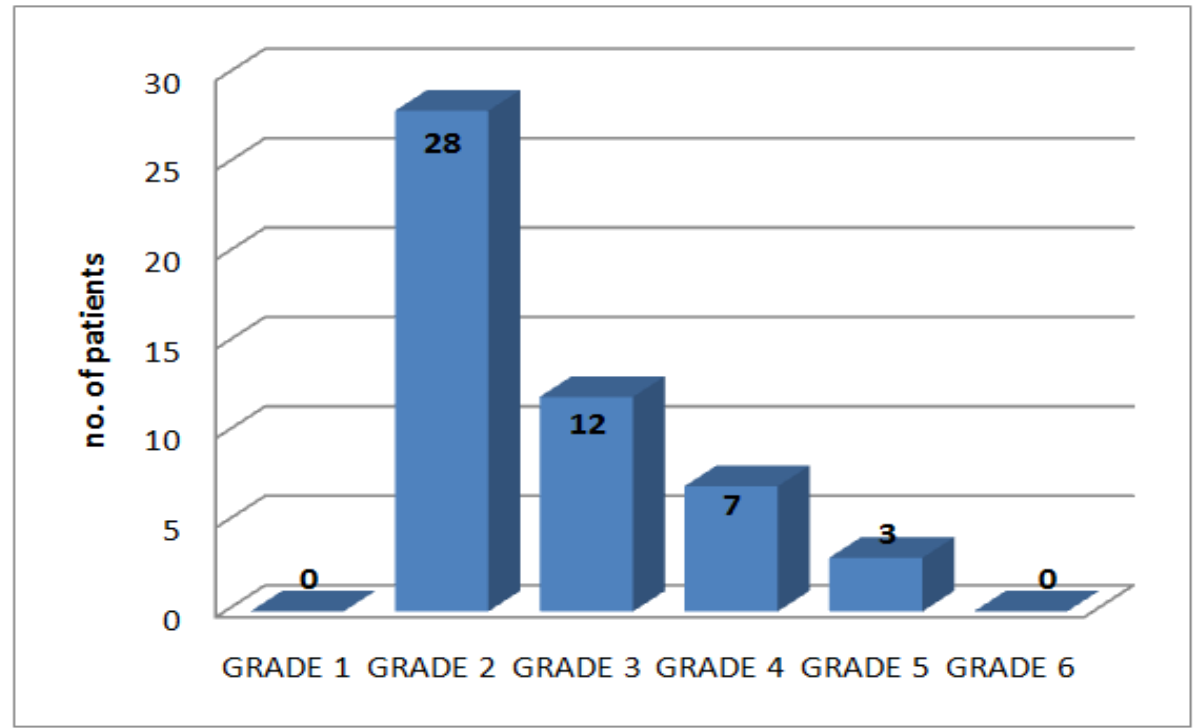

Figure 4 - Bar chart showing grades after 6 months 


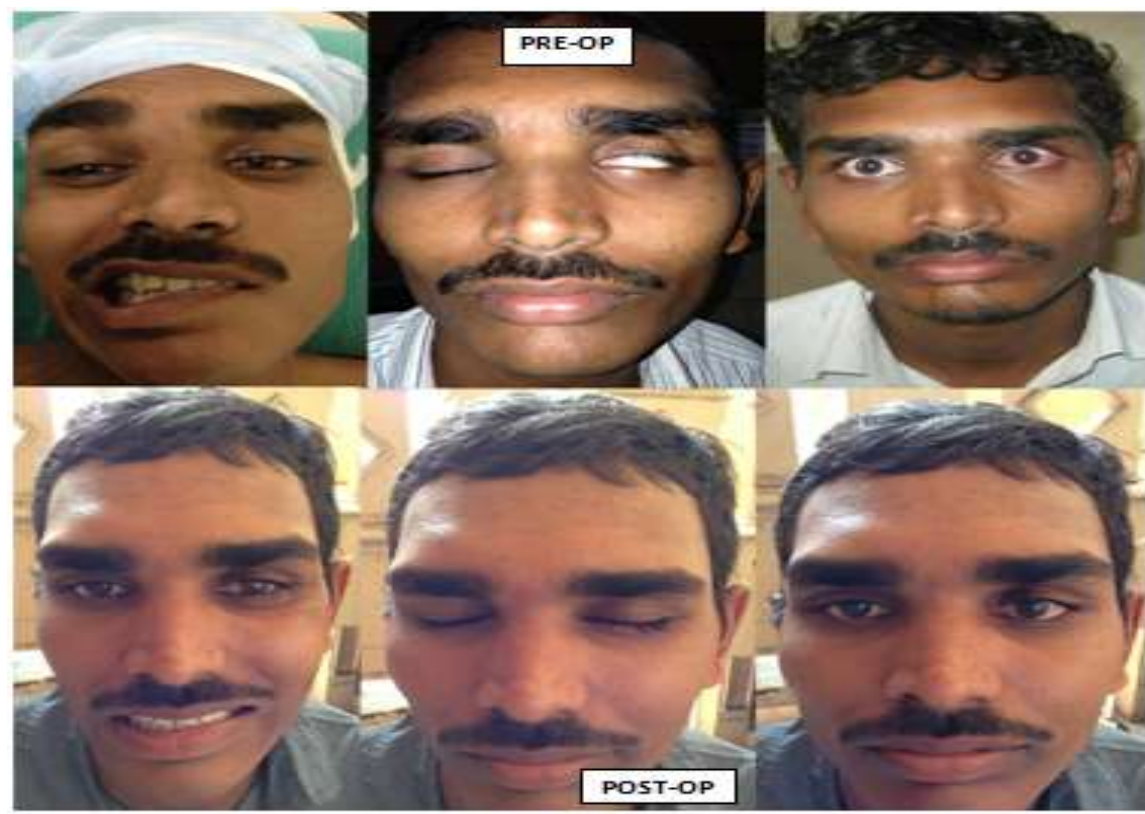

